Mercadé Melé, Molinillo Jiménez y Fernández Morales(2014) / Revista de Empresa Familiar, 4(1), 73-88. www.revistadeempresafamiliar.uma.es

\title{
Influencia de las prácticas de responsabilidad social corporativa en la actitud del consumidor: análisis comparado de Mercadona, Carrefour y Eroski \\ Influence of the corporate social responsibility practices in the consumer attitude: a comparative analysis between Mercadona, Carrefour and Eroski
}

\author{
Pere Mercadé-Mele ${ }^{\mathrm{a}^{*}} \cdot$ Sebastián Molinillo-Jiménez ${ }^{\mathrm{b}} \cdot$ Antonio Fernández-Morales $^{\mathrm{a}}$ \\ ${ }^{a}$ Departamento de Economía Aplicada, Universidad de Málaga (Málaga) \\ ${ }^{b}$ Departamento de Organización de Empresas y Finanzas, Universidad de Málaga (Málaga)
}

D A T OS A R T ÍC U L O

\section{Historial:}

Recibido 15-12-2013

Aceptado 05-04-2014

Palabras clave:

Responsabilidad Social

Corporativa

Modelo de Desarrollo

Sostenible

Actitud del consumidor

Empresa Familiar

Minorista

Códigos JEL: M14
R E S U M E N

La implantación de prácticas de Responsabilidad Social Corporativa (RSC) en las empresas es un tema relevante en los últimos años. Entre las cuestiones analizadas destaca la importancia de correlacionar la RSC con el comportamiento de los clientes por la influencia que puede tener en los beneficios de las empresas. Si bien diferentes investigaciones han estudiado esta relación previamente, son pocos los trabajos que han tratado la influencia de la RSC percibida en la actitud del consumidor en el sector de la distribución comercial minorista de España y menos aún en un contexto de crisis económica.

En esta investigación se analiza la relación entre la RSC percibida y la actitud del consumidor de los tres grupos empresariales del sector minorista con mayor facturación en España: la empresa familiar Mercadona y las enseñas Carrefour y Eroski.

Los resultados corroboran que las acciones de RSC influyen de manera directa y positiva en la actitud del consumidor, por lo que la investigación contribuye a consolidar el conocimiento de la relación entre estas dos variables y orienta a los gestores hacia la implantación de políticas de RSC para mejorar sus resultados.

\section{A R T I C LE IN F O \\ Article history: \\ Received 15-12-2013 \\ Accepted 05-04-2014}

Keywords:

Corporate Social

Responsibility

Sustainable Development

Model

Consumer Attitude

Family Business

Retail

JEL codes: M14

\begin{abstract}
A B S T R A C T
The practical implementation of Corporate Social Responsibility (CSR) in business has been an important issue in the recent years. Among the issues discussed, it is valuable to highlight the importance of CSR correlated with the customer behaviour which is related to the company profit.

Although various studies have examined this relationship in the past, there were few researches dedicated to the perceived influence of CSR on a consumer attitude in the field of retail distribution in Spain, and particularly in the current context of economical crisis.

This research examines the relationship between perceived CSR and consumer attitudes of three business groups with the highest retail sales in Spain: the Mercadona family business and the companies Carrefour and Eroski.

The results confirm that CSR actions have a direct and positive impact on consumer attitudes; hence this research contributes to consolidate the knowledge of the relationship between these two variables and provide guidance to managers to implement CSR policy to improve their results.
\end{abstract}

\footnotetext{
*Autor de contacto.

Correos electrónicos: pmercade@uma.es, smolinillo@,uma.es, afdez@,uma.es
} 


\section{Introducción}

La Responsabilidad Social Corporativa (RSC) es una forma de gestión de los negocios que en los últimos años está atrayendo una gran atención por parte de los académicos debido al interés por determinar cómo contribuye a la mejora de la sociedad y a la competividad de las empresas (Porter y Kramer, 2011). En este sentido, la diversidad de las respuestas de los consumidores ante las iniciativas de RSC hace necesario profundizar en el conocimiento de su comportamiento (Sen y Bhattacharya, 2001).

El informe de MORI (2000) sostiene que el 70\% de los consumidores europeos consideran importante los criterios de la RSC a la hora de adquirir un producto o servicio y el $20 \%$ estaría dispuesto a pagar un sobreprecio. Por otra parte, en el trabajo de CONE (2002) se pone de manifiesto una influencia aún mayor de la RSC en el comportamiento del consumidor, dado que en EEUU el 84\% estaría dispuesto a cambiar su marca habitual por otra que realizase actividades sociales si el precio y la calidad fuesen parecidos.

En el sector comercial minorista, según Arnold et al. (1996), los consumidores perciben la imagen de la tienda no sólo a través de los atributos tradicionales (instalaciones, comodidad, precio, variedad,...) sino también mediante el papel de la tienda en un entorno social más amplio. Handelman y Arnold (1999) consideran que los consumidores apoyan al minorista que realiza acciones socialmente responsables, lo que implica una mayor intención de compra en las tiendas o un aumento de la comunicación "boca a oreja" sobre la actividad minorista. Por lo tanto, parece lógico que la empresa comercial minorista se caracterice por realizar fuertes inversiones en programas de RSC (Barone et al., 2000; Gupta y Pirsch, 2008).

Sin embargo, aunque diferentes estudios hayan demostrado una relación positiva entre las acciones sociales y el comportamiento del mercado hacia la organización (Du et al., 2007; Bhattacharya et al., 2008; Hildebrand et al., 2011), en España aún no se ha observado un claro posicionamiento de las empresas hacia la implantación de modelos de gestión que consideren prioritarias las políticas de la RSC (IPSOS, 2011). Además, la crisis económica ha suscitado un creciente interés en la RSC por parte de las empresas debido a las mayores exigencias de la sociedad (Pérez y Rodríguez, 2012), a la mayor resiliencia de las actividades de RSC y a la necesidad de esclarecer su impacto en las decisiones de compra, en un momento en el que diversos investigadores consideran que la RSC será de las primeras políticas de las que las empresas van a prescindir ((Harwood et al., 2011).

Las políticas de RSC que realizan las empresas puede tener diferentes interpretaciones en diferentes países, para diferentes personas y en distintos momentos (Campbell, 2007; Peloza y Shang, 2011). En el caso de empresas minoristas, el comportamiento del consumidor ante acciones de RSC ha sido estudiado en países como: EEUU (Gupta y Pirsch, 2008), Reino Unido (Oppewal, 2006), Corea (Ko et al., 2013), Italia (Massa y Testa, 2012) o Suecia (Anselmsson y Johansson, 2007), así como comparaciones entre países como EEUU y Alemania (Walsh y Bartikowski; 2013) o Brasil y Francia (Barin y Michael, 2010). En el caso de España Carrero y Valor (2012) han realizado un estudio de la RSC en el sector minorista; estos autores analizaron el etiquetado de productos de ocho minoristas de España y Reino Unido para ver si los consumidores eran capaces de hacer una cesta de la compra ética pero no analizaron las relaciones entre la RSC percibida y el comportamiento de compra hacia esos minoristas, como otros trabajos han tratado de poner de manifiesto (Ko et al., 2013; Wan y Schell, 2007). El estudio de estas relaciones es fundamental para las empresas, porque la diferenciación de la oferta en base a atributos clásicos como el precio o la calidad es cada vez más difícil (Marín y Ruiz, 2007), mientras que por el contrario la RSC se puede convertir en un importante instrumento de diferenciación competitiva (Drumwright, 1996; Brammer y Millington, 2005; Du et al., 2007; Maignan et al., 2005; Bhattacharya y Sen, 2003).

Por lo tanto, en este trabajo se pretende atender el vacío que existe en España en torno al estudio de las relaciones de la RSC percibida por el consumidor con su actitud hacia la empresa minorista, considerando las posibles diferencias entre la empresa familiar Mercadona y las sociedades Carrefour y Eroski.

Con este objetivo el documento se ha estructurado de la siguiente manera: en primer lugar se revisa la literatura en torno a los conceptos de RSC, su importancia en el sector de la distribución comercial minorista y, en particular, en la empresa familiar Mercadona, los componentes de la actitud del consumidor y se establece el modelo teórico de relación entre estos componentes y la RSC; a continuación se describe la metodología con indicación de las escalas de medida utilizadas así como los procedimientos de muestreo y recogida de 
datos; posteriormente se analizan los resultados de la investigación empírica y finalmente se comentan las principales conclusiones e implicaciones para los gerentes de las empresas, así como se proponen futuras líneas de investigación.

\section{Revisión de la literatura}

\subsection{La Responsabilidad Social Corporativa Percibida}

El concepto de la RSC presenta múltiples y diferentes definiciones (Carroll, 1999; Garriga y Mele, 2004; Maignan y Ferrell, 2004; Göbbels, 2002). Esta indefinición provoca una ambigüedad en su conceptualización que deriva en dificultades para conocer cómo los clientes forman sus juicios sobre la RSC (Peloza y Shang, 2011). De igual modo, tampoco hay acuerdo sobre las dimensiones que constituyen la RSC percibida del consumidor (Maignan, 2001; García de Salmones et al., 2005; Van Marrewijk, 2003; Bigné et al., 2012), por lo que en este trabajo se adopta el marco teórico definido por el enfoque de Desarrollo Sostenible, dado que es uno de los más utilizados para explicar casos de éxitos empresariales (Savitz, 2013). Este enfoque fue formulado originariamente por John Elkington para quien la responsabilidad corporativa del siglo XXI es fundamental para progresar hacia la prosperidad económica, la calidad ambiental y la justicia social, denominándolo "the triple bottom line" o "People, Planet y Profit" (Elkington, 1997, 1998; Jeurissen, 2000; Wheeler y Elkington, 2001; Norman y Macdonald, 2004). Por lo tanto, este enfoque tiene en cuenta las preocupaciones sociales, medioambientales y económicas a través de las personas, la sociedad y los beneficios (Bigné et al. 2005; Slaper y Hall, 2011; Balmer, 2011; Powell, 2011).

\subsection{La RSC en el sector minorista}

Las empresas del sector minorista pretenden que las relaciones con sus clientes sean estables y duraderas (Grönroos, 1994; Arnett et al., 2003; Marín y Ruiz, 2007; Bhattacharya et al., 2008). En este sentido, la cada vez mayor importancia que los consumidores prestan al comportamiento ético de las empresas del sector (Carrigan y Attalla, 2001; Nicholls, 2002; Anselmsson y Johansson, 2007; Auger y Devinney, 2007) ha generado un aumento de los fondos, tiempo y esfuerzos dedicados a las actividades de RSC con el fin de influir en las percepciones generales de los consumidores (Gupta y Pirsch, 2008). Este comportamiento de las empresas y de los consumidores ha propiciado que el sector se haya convertido en uno de los focos de atención de la investigación reciente sobre la RSC (Kilcullen y Kooistra, 1999; Nicholls, 2002; Adams, 2002; Oppewal et al., 2006; Gupta y Pirsch, 2008; Walsh y Bartikowski, 2013).

Una de las características del sector es que un número reducido de empresas acaparan un volumen importante de las ventas a nivel mundial (Deloitte, 2013), alcanzando extremos tales como que en España solo las cuatro mayores empresas de alimentación ostenten el $58 \%$ de la cuota de mercado (Secretaría de Estado de Comercio, 2011). Esta posición en el mercado es observada por los consumidores como depredadora y alejada de sólidas prácticas de RSC (Connor, 2001), por lo que sus acciones sociales suelen percibirse como egoístas (Torres et al., 2012) y tienen un menor efecto positivo sobre la empresa (Prout, 2006; Yoon et al., 2006). Por lo tanto, los minoristas son conocedores de que cualquier comportamiento no ético puede dañar a la empresa y destruir su imagen (Fan, 2005), por lo que no solo están prestando atención a sus propias políticas, sino que además están demandando un comportamiento más ético y responsable por parte de sus proveedores (Grunert, 2005; Massa y Testa, 2012).

Por otra parte, en la expansión internacional de los minoristas sus relaciones con los consumidores pueden variar según el país y la cultura (Walsh y Bartikowski, 2013) y estas diferencias tienen influencias en la formación de la satisfacción y del comportamiento del consumidor (Laroche et al., 2004; Morgeson et al., 2011). Según, Walsh y Bartikowski (2013) hasta ahora pocas investigaciones han abordado estas diferencias culturales en los antecedentes de la actitud de los consumidores lo que dificulta la aplicación de estrategias globales en la internacionalización de las empresas.

Por lo tanto, en este trabajo se analizarán las relaciones de la RSC percibida por el consumidor con su actitud hacia la empresa minorista de España $\mathrm{y}$, concretamente, hacia los tres mayores grupos de distribución alimentaria: Mercadona, Carrefour, y Eroski (INDISA, 2011). Estas empresas ocupan el puesto $2^{\circ}, 36^{\circ}$ y $48^{\circ}$, respectivamente, en el ranking de Empresas Responsables (Merco, 2013).

\subsection{La RSC en la empresa familiar: el caso Mercadona}

La empresa familiar está cada vez más presente en el debate sobre la RSC (Murillo y Lozano, 2006; 
Jamali et al., 2009). Si bien Jenkins (2006) considera que la naturaleza de las relaciones entre los grupos de interés no puede ser drásticamente diferentes, si es probable que se diferencien en la gestión. Miller et al. (2008) considera que en las empresas familiares esta diferencia radica en que los grupos de interés que cobran más fuerza son los empleados y los clientes, para garantizar su continuidad en el tiempo. Vives (2006) destaca la influencia de los gerentes y de los propietarios en las acciones de las empresas familiares, donde la ética y los valores morales de éstos marcan el matiz de la RSC de las empresas. Además, en las empresas familiares existe una sensibilidad social y cultural acorde con los valores y normas de los propietarios (Dyer, 1988; Hall y Nordqvist, 2008; Fassin et al., 2011).

Una de las empresas familiares más importantes de España por número de empleados y volumen de facturación es Mercadona (Fernández, 2013). Según su Memoria Anual de 2013 es una compañía de distribución de capital $100 \%$ español y familiar, su presidente ejecutivo es Juan Roig, está presente en el mercado nacional con 1.467 supermercados, 74.000 empleados con contrato fijo, una cuota de mercado en superficie total de venta del $14,1 \%$, una facturación de 19.812 millones de euros y compras en España por valor de 14.500 millones de euros y una aportación al PIB nacional de 3.700 millones de euros, lo que representa un $4 \%$ más que en el ejercicio 2012 (Mercadona, 2014).

Si bien Mercadona no presenta un informe sobre la RSC su Memoria Anual hace referencia a los tres componentes del modelo de Desarrollo Sostenible. En este sentido, una encuesta realizada por el
Instituto Opinea (2013) para evaluar el impacto que las acciones de RSC realizadas por 65 grandes empresas tienen sobre su imagen corporativa se considera a Mercadona como una de las empresas con mejor valoración en los tres aspectos considerados: medioambiental, social y humanitario.

\subsection{La actitud del consumidor}

La actitud tiene un papel primordial en el comportamiento del consumidor y en la decisión de compra (Bagozzi y Dholakia, 1999). Según diversos autores (Bagozzi, 1997; Krech et al., 1962; Rosenberg y Hovland, 1960; Alonso y Grande, 2010; Rivera et al., 2009) la actitud se define a partir de tres componentes: cognitivo, afectivo y conductual.

\section{Tabla 1}

Elementos que componen la actitud.

\begin{tabular}{cc}
\hline Componente & Manifestación \\
\hline \multirow{3}{*}{ Cognitivo } & Opiniones \\
& $\begin{array}{c}\text { Experiencias } \\
\text { Creencias }\end{array}$ \\
\hline \multirow{3}{*}{ Afectivo } & Emociones \\
& Sentimientos \\
& Valoraciones \\
\hline \multirow{3}{*}{ De conducta } & Intención de compra \\
& Respuesta de compra \\
& Respuesta de rechazo \\
\hline
\end{tabular}

Fuente: Krech et al., 1962; Bagozzi et al., 1979; Alonso y Grande, 2010.

El componente afectivo y el cognitivo dan origen a la satisfacción del consumidor (Swaen y Trawick,

\section{Figura 1}

Modelo teórico propuesto.

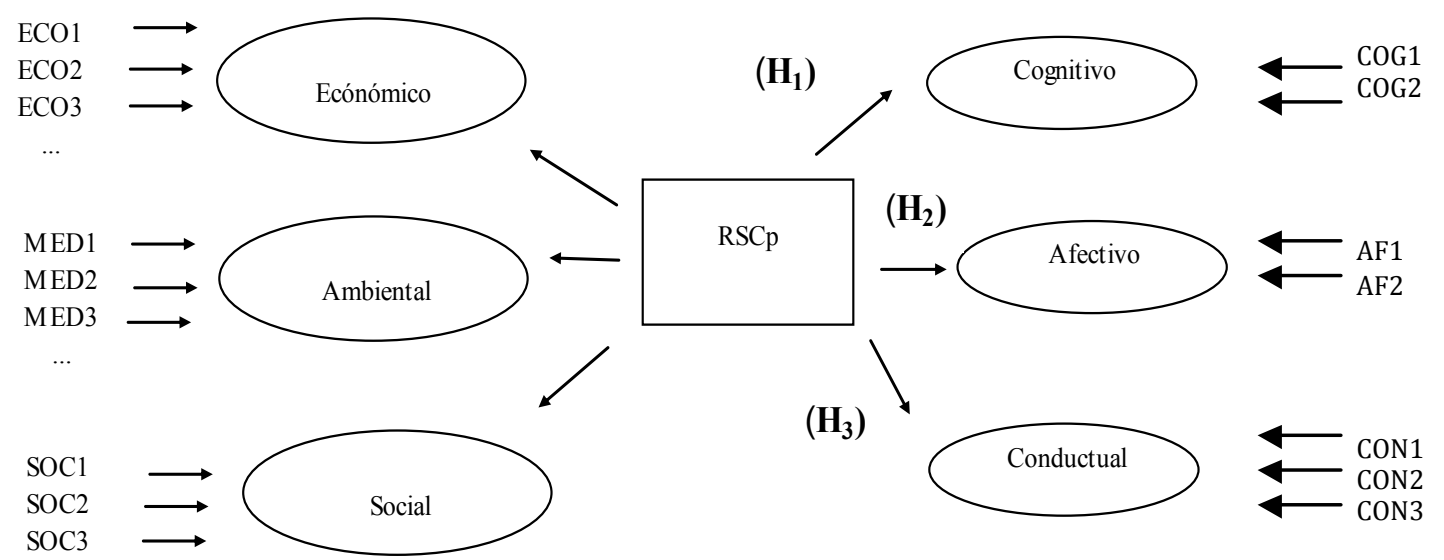


1981; Oliver et al., 1997; Cronin et al., 2000; Taylor et al., 2007; Wirtz y Bateson, 1999; Bigné et al., 2011) que se define como una evaluación general basada en la compra del consumidor y en la experiencia de consumo (Anderson et al., 2004). El componente cognitivo se refiere a las propias creencias, a la información y a los conocimientos del producto en cuestión. El componente afectivo recoge los sentimientos y las emociones que tiene una persona hacia un producto, empresa o persona.

Finalmente, el componente de conducta o comportamiento es la predisposición a la acción que experimenta el individuo (véase tabla 1 ).

\subsection{Relación de la RSC percibida con la actitud del consumidor}

Una trayectoria sólida en la política de RSC creara un contexto favorable que impulsara una valoración positiva de los consumidores y generara una buena actitud hacia la empresa (Brown y Dacin,
1997; Gürhan-Canli y Batra, 2004; Sen y Bhattacharya, 2001; Peloza y Shang, 2011).

La RSC se relaciona directamente con la actitud de los consumidores ya que influye en que estos: tengan una imagen positiva de la empresa (Simon, 1995; Berens et al., 2005), realicen una mejor valoración de sus productos (Brown y Dacin, 1997; Brown, 1998), presten un mayor apoyo a la organización (Handelman y Arnold, 1999) y presenten una mejor predisposición hacia su oferta (Berens et al., 2005).

Además, la RSC influye en: la satisfacción del consumidor (Luo y Bhattacharya, 2006; Bigné et al., 2011; Green y Peloza, 2011), en la intención de compra (Sen y Bhattacharya, 2001; Bigné y Currás, 2008) y en la lealtad (Pérez et al., 2012; Ko et al., 2013).

Por lo tanto, se pueden enunciar las siguientes hipótesis: H1: La RSC percibida influye directa $y$ positivamente en el componente cognitivo de la

\section{Tabla 2}

Ítems para la medición de la dimensión económica, medioambiental y social de las percepciones de la RSC.

\begin{tabular}{|c|c|c|}
\hline Dimensión & Ítems & Investigaciones \\
\hline \multirow{3}{*}{ Económica } & ECO1 Le preocupa fidelizar a sus clientes & $\begin{array}{l}\text { (Sirgy et al. (1991); Reichheld (2003); Kamins y } \\
\text { Folkes (1999); Becker-Olsen et al. (2006) }\end{array}$ \\
\hline & $\begin{array}{l}\text { ECO2 Le preocupa la calidad de sus } \\
\text { productos }\end{array}$ & Bigné et al. $(2005,2006)$ \\
\hline & $\begin{array}{l}\text { ECO3 Le preocupa que los trabajadores } \\
\text { estén bien remunerados }\end{array}$ & $\begin{array}{l}\text { Boal y Peery (1986); Maignan et al. (1999); Sen et al. } \\
\text { (2006) }\end{array}$ \\
\hline \multirow{5}{*}{ Medioambiental } & $\begin{array}{l}\text { MED1 Patrocina actividades a favor del } \\
\text { medioambiente }\end{array}$ & $\begin{array}{l}\text { Maignan et al. (1999); Bigné et al. (2005, 2006); } \\
\text { García de los Salmones et al. (2005) }\end{array}$ \\
\hline & $\begin{array}{l}\text { MED2 Reduce el despilfarro de recursos } \\
\text { (energía, materias primas,...) }\end{array}$ & $\begin{array}{l}\text { Brown y Dacin (1997); Sen y Bhattacharya (2001); } \\
\text { Bigné et al. }(2005,2006) \text {; Fernández y Merino (2005) }\end{array}$ \\
\hline & $\begin{array}{l}\text { MED3 Publica un informe anual del } \\
\text { impacto medioambiental de su actividad }\end{array}$ & Bigné et al. $(2005,2006)$ \\
\hline & $\begin{array}{l}\text { MED4 Invierte en I+D para mejorar su } \\
\text { actividad a favor del medioambiente }\end{array}$ & Bigné et al. $(2005,2006)$ \\
\hline & MED5 Recicla adecuadamente sus residuos & $\begin{array}{l}\text { Brown y Dacin (1997); Sen y Bhattacharya (2001); } \\
\text { Fernández y Merino (2005); Menon y Kahn (2003) }\end{array}$ \\
\hline \multirow{5}{*}{ Social } & $\begin{array}{l}\text { SOC1 Intenta tener un trato justo a sus } \\
\text { trabajadores con independencia de sexo, } \\
\text { raza o religión }\end{array}$ & $\begin{array}{l}\text { Maignan (2001); Bigné et al. }(2005,2006) \text {; Sen et al. } \\
(2001,2006)\end{array}$ \\
\hline & SOC2 Ayuda a países en desarrollo & $\begin{array}{l}\text { Bigné et al. (2005, 2006); Fernández y Merino } \\
\text { (2005); García de los Salmones et al. (2005) }\end{array}$ \\
\hline & $\begin{array}{l}\text { SOC3 Realiza donaciones económicas a } \\
\text { causas sociales }\end{array}$ & Brown y Dacin (1997), Bigné et al. (2011) \\
\hline & $\begin{array}{l}\text { SOC4 Intenta tener prácticas igualitarias de } \\
\text { oportunidades de empleo }\end{array}$ & $\begin{array}{l}\text { Brown y Dacin (1997); Maignan (2001); Sen y } \\
\text { Bhattacharya (2001); Bigné et al. (2005, 2006); } \\
\text { Fernández y Merino (2005); Sen et al. (2006) }\end{array}$ \\
\hline & $\begin{array}{l}\text { SOC5 Le preocupa la creación de puestos } \\
\text { de trabajo }\end{array}$ & Bigné et al. $(2005,2006)$ \\
\hline
\end{tabular}


actitud del consumidor. H2: La RSC percibida influye directa y positivamente en el componente afectivo de la actitud del consumidor.

La intención de compra de los consumidores se correlaciona positivamente con el grado de valoración de la percepción de la RSC (Creyer y Ross, 1997). En este sentido, los consumidores apoyan al minorista que realiza acciones socialmente del consumidor se ha construido un conjunto de tres factores de primer orden (cognitivo, afectivo y conductual) a partir de siete variables observadas. Los indicadores que forman estas dimensiones son reflectivos porque son manifestaciones del constructo, comparten un tema común, se espera que sean intercambiables y que estén fuertemente correlacionados (Chin y Tood, 1995; Mathieson et

Tabla 3

Ítems para la medición de la actitud a partir de sus componentes cognitivo, afectivo y de conducta.

\begin{tabular}{|c|c|c|}
\hline Componente & Ítems & Investigaciones \\
\hline \multirow[t]{2}{*}{ Cognitivo } & $\begin{array}{l}\text { COG1 Proporciona } \\
\text { información acerca de sus } \\
\text { productos }\end{array}$ & Wuu y Petroshius (1987) \\
\hline & $\begin{array}{l}\text { COG2 Me merece una } \\
\text { opinión favorable }\end{array}$ & $\begin{array}{l}\text { Wuu y Petroshius (1987); Fullerton (2003); Sen et } \\
\text { al. (2006); Cronin et al. (2000); Berry (2000); } \\
\text { Keller (1993) }\end{array}$ \\
\hline \multirow[t]{2}{*}{ Afectivo } & $\begin{array}{l}\text { AFE1 Proporciona una } \\
\text { experiencia gratificante }\end{array}$ & Cronin et al (2000); Brown y Dacin (1997) \\
\hline & AFE2 Me inspira confianza & $\begin{array}{l}\text { Bergami y Bagozzi (2000); Fullerton (2003); Sen } \\
\text { et al. (2006) }\end{array}$ \\
\hline \multirow[t]{3}{*}{ Conductual } & $\begin{array}{l}\text { CON1 Es el centro donde voy } \\
\text { a realizar la próxima compra }\end{array}$ & Putrevu y Lord (1994); Sen et al. $(2001,2006)$ \\
\hline & $\begin{array}{l}\text { CON2 Es el centro que voy a } \\
\text { recomendar para hacer las } \\
\text { compras }\end{array}$ & $\begin{array}{l}\text { Sirgy et al. (1991); Reicheld (2003); Zeithaml et } \\
\text { al. (1996); Kamins y Folkes (1999); Söderlund } \\
\text { (2002) }\end{array}$ \\
\hline & $\begin{array}{l}\text { CON3 Es el centro al que le } \\
\text { voy ser fiel }\end{array}$ & $\begin{array}{l}\text { Sirgy et al. (1991); Reicheld (2003); Kamins y } \\
\text { Folkes (1999) }\end{array}$ \\
\hline
\end{tabular}

responsables con una mayor intención de compra en sus tiendas (Arnold, et al., 1996) y en otros sectores diferentes investigaciones han puesto de manifiesto una relación positiva entre la RSC y la intención de compra (Du et al., 2007; Sen y Bhattacharya, 2001; Bigné et al., 2005, 2006). Consecuentemente, se enuncia la siguiente hipótesis: H3: La RSC percibida influye directa y positivamente en el componente conductual de la actitud del consumidor.

Para contrastar las hipótesis de este trabajo se ha diseñado un modelo estructural con variables latentes (véase figura 1).

En el modelo la RSC percibida es un factor no observado de segundo orden, cuyos factores de primer orden se corresponden con las tres dimensiones del enfoque de Desarrollo Sostenible (económica, ambiental y social) construidas a su vez a partir de trece variables observadas. Por otra parte, para evaluar la relación directa de la RSC percibida con cada uno de los tres componentes de la actitud al., 2001; Mackenzie et al., 2005), de la misma manera que en otros trabajos previos (Luo y Bhattacharya, 2001; Perez et al., 2012; Sen y Bhattacharya, 2001; Bigné et al., 2012; Ko et al., 2013).

\section{Metodología}

\subsection{Escalas de medida}

Para establecer y validar la relación entre la RSC percibida y la actitud del consumidor se ha realizado una encuesta, en la que se le pedía a los participantes que indicaran como valoraban su percepción de una serie de afirmaciones sobre las prácticas socialmente responsables de las empresas consideradas así como su grado de acuerdo con afirmaciones que describen su actitud hacia esas empresas. La escala de medición que se ha utilizado es una escala aditiva de 
Figura 3

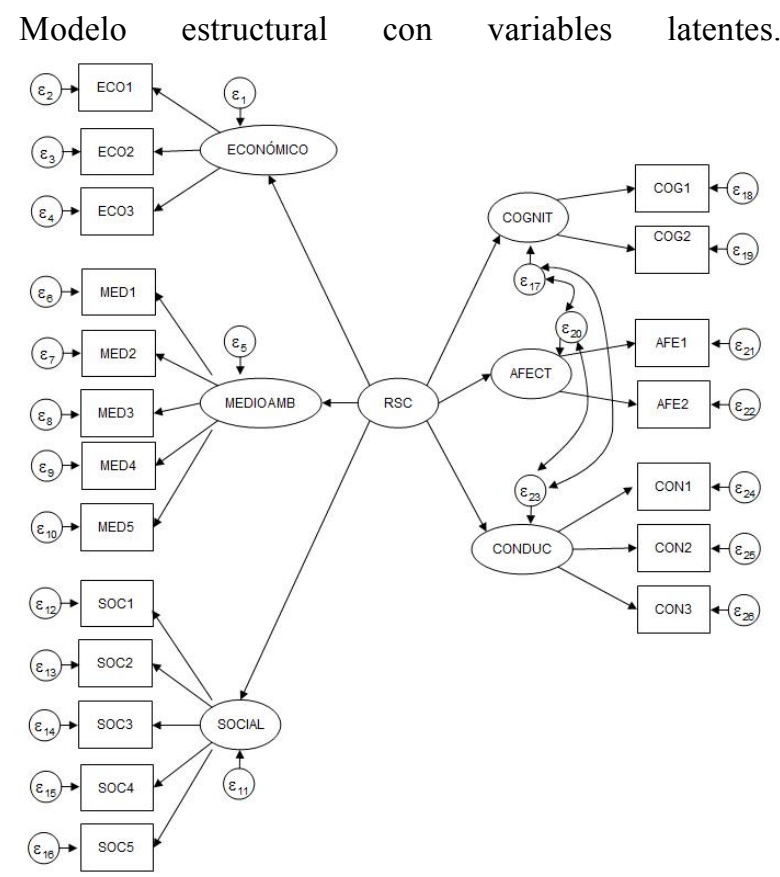

Likert, de 1 (Totalmente en desacuerdo) a 7 (Totalmente de acuerdo).

La revisión de la literatura científica ha permitido establecer catorce ítems para medir las tres dimensiones del enfoque de Desarrollo Sostenible: tres de ellos para medir la dimensión económica, cinco para la dimensión medioambiental y otros cinco para la dimensión social (véase tabla 2).

Por otra parte, para medir la actitud se han establecido siete ítems: dos en el componente cognitivo, otros dos para el componente afectivo y tres para el componente conductual o intención de compra (véase tabla 3 ).

\subsection{Muestra y recogida de datos}

En la selección de la muestra se ha considerado que los estudiantes universitarios son jóvenes consumidores que formaran parte de los futuros grupos de dirigentes sociales y empresariales, por lo que su opinión puede aportar indicios sobre las pautas del comportamiento de los futuros consumidores (Bigné et al., 2005). Por lo tanto, de acuerdo con Guiry et al. (2006) y Wagner et al. (2008), los datos del análisis proceden de una muestra de conveniencia de 235 estudiantes de grado matriculados en asignaturas del área de marketing que conocen las empresas propuestas y que son clientes habituales de las mismas (Walsh y Bartikowski; 2013) por lo que respondieron a la totalidad de las cuestiones planteadas.

La investigación se realizó en el contexto del comercio minorista porque, como se ha indicado, las empresas de este sector realizan importantes inversiones en RSC (Barone et al., 2000; Gupta y Pirsch, 2008). En este sentido, las cuestiones se plantearon sobre empresas del sector de alimentación por considerar que las compras son más frecuentes que en otros sectores y, por lo tanto, el contacto consumidor-empresa es más habitual. Los encuestados evaluaron las tres empresas de distribución alimentaria con mayor facturación en España según Indisa (2011): Mercadona, Carrefour y Eroski. Además, estas empresas son las tres primeras del sector de alimentación incluidas en el ranking de Empresas Responsables (Merco, 2013) y publican en su página web la Memoria Anual que incluye las acciones de responsabilidad social, práctica que solo siguen el $67 \%$ de las grandes empresas detallistas del mundo (Baker et al., 2012).

\section{Tabla 4}

Medidas de fiabilidad y validez convergente.

\begin{tabular}{lllllllllll}
\hline \multicolumn{1}{c}{ Cronbach's a } & \multicolumn{7}{c}{ Composite reliability index $\mathrm{r}_{\mathrm{h}}$} & AVE \\
\hline & Mercadona & Carrefour & Eroski & Mercadona & Carrefour & Eroski & Mercadona & Carrefour Eroski \\
Dimensión & & & & & & & & & & \\
Económica & 0,7008 & 0,7017 & 0,7110 & 0,8353 & 0,8368 & 0,7957 & 0,6285 & 0,6313 & 0,5654 \\
Ambiental & 0,8413 & 0,8039 & 0,8003 & 0,8884 & 0,8659 & 0,8693 & 0,6148 & 0,5639 & 0,5715 \\
Social & 0,8000 & 0,8127 & 0,8082 & 0,8625 & 0,8700 & 0,8700 & 0,5581 & 0,5729 & 0,5735 \\
Componente & & & & & & & & & & \\
Cognitivo & 0,7165 & 0,7164 & 0,7132 & 0,8541 & 0,8511 & 0,8451 & 0,7453 & 0,7408 & 0,7317 \\
Afectivo & 0,8174 & 0,7831 & 0,7812 & 0,9152 & 0,9026 & 0,9019 & 0,8436 & 0,8225 & 0,8214 \\
Conductual & 0,8308 & 0,8337 & 0,8402 & 0,9006 & 0,9003 & 0,9035 & 0,7516 & 0,7506 & 0,7573 \\
\hline
\end{tabular}




\section{Análisis de datos}

Previamente a la estimación del modelo propuesto se ha evaluado la fiabilidad de las escalas de medición. Respecto a la consistencia interna de las dimensiones de la RSC se ha utilizado el coeficiente $\alpha$ de Cronbach y el índice de fiabilidad compuesta, $\rho \eta$ (composite reliability index, CRI) (Fornell y Larcker, 1981). Tanto los coeficientes $\alpha$ de Cronbach como los valores del índice de fiabilidad compuesta son superiores a 0,7 , lo que indica una adecuada fiabilidad de los constructos en lo relativo a la consistencia interna de los mismos (véase Tabla 4).

\section{Tabla 5}

Medidas de validez discriminante.

\begin{tabular}{|c|c|c|c|c|c|c|}
\hline \multicolumn{7}{|l|}{ Mercadona } \\
\hline & Económica & Ambiental & Social & Cognitivo & Afectivo & Conductual \\
\hline Económica & 0,6285 & & & & & \\
\hline Ambiental & 0,1333 & 0,6148 & & & & \\
\hline Social & 0,2099 & 0,4193 & 0,5581 & & & \\
\hline Cognitivo & 0,2951 & 0,1590 & 0,3951 & 0,7453 & & \\
\hline Afectivo & 0,2591 & 0,0829 & 0,2449 & 0,5229 & 0,8436 & \\
\hline Conductual & 0,1827 & 0,0601 & 0,1839 & 0,3741 & 0,5298 & 0,7516 \\
\hline \multicolumn{7}{|l|}{ Carrefour } \\
\hline & Económica & Ambiental & Social & Cognitivo & Afectivo & Conductual \\
\hline Económica & 0,6313 & & & & & \\
\hline Ambiental & 0,0985 & 0,5639 & & & & \\
\hline Social & 0,1080 & 0,3717 & 0,5729 & & & \\
\hline Cognitivo & 0,2562 & 0,1475 & 0,3171 & 0,7408 & & \\
\hline Afectivo & 0,1751 & 0,0916 & 0,1663 & 0,4797 & 0,8225 & \\
\hline Conductual & 0,0423 & 0,0979 & 0,0869 & 0,1955 & 0,3217 & 0,7506 \\
\hline
\end{tabular}

Por otra parte, se ha evaluado la validez convergente de los constructos de primer orden utilizando la varianza media extraída (average variance extracted, AVE), (Fornell y Larcker, 1981). En todos los casos el valor de la varianza media extraída es superior al valor mínimo de 0,5. Además, todas las cargas factoriales estandarizadas son superiores a 0,7 (Hair et al., 2010).

Por último, siguiendo a Fornell y Larcker (1981), se ha evaluado la validez discriminante de los constructos de primer orden comprobando que la varianza media extraída para cada constructo es superior a la correlación con los restantes

.

\begin{tabular}{lllllll}
\hline Eroski & & & & & \\
& Económica & Ambiental & Social & Cognitivo & Afectivo & Conductual \\
Económica & 0,5654 & & & & & \\
Ambiental & 0,1530 & 0,5715 & & & & \\
Social & 0,1661 & 0,4307 & 0,5735 & & & \\
Cognitivo & 0,3429 & 0,1556 & 0,3480 & 0,7317 & & \\
Afectivo & 0,2618 & 0,0838 & 0,2192 & 0,5339 & 0,8214 & 0,7573 \\
Conductual & 0,1449 & 0,0517 & 0,0905 & 0,2073 & 0,2859 & \\
& \\
Mercadé Melé, P., Molinillo Jiménez S. y Fernández Morales, A.(2014). Influencia de las prácticas de responsabilidad social corporativa \\
en la actitud del consumidor: análisis comparado de Mercadona, Carrefour y Eroski. Revista de Empresa Familiar, 4 (1), 73-88.
\end{tabular}




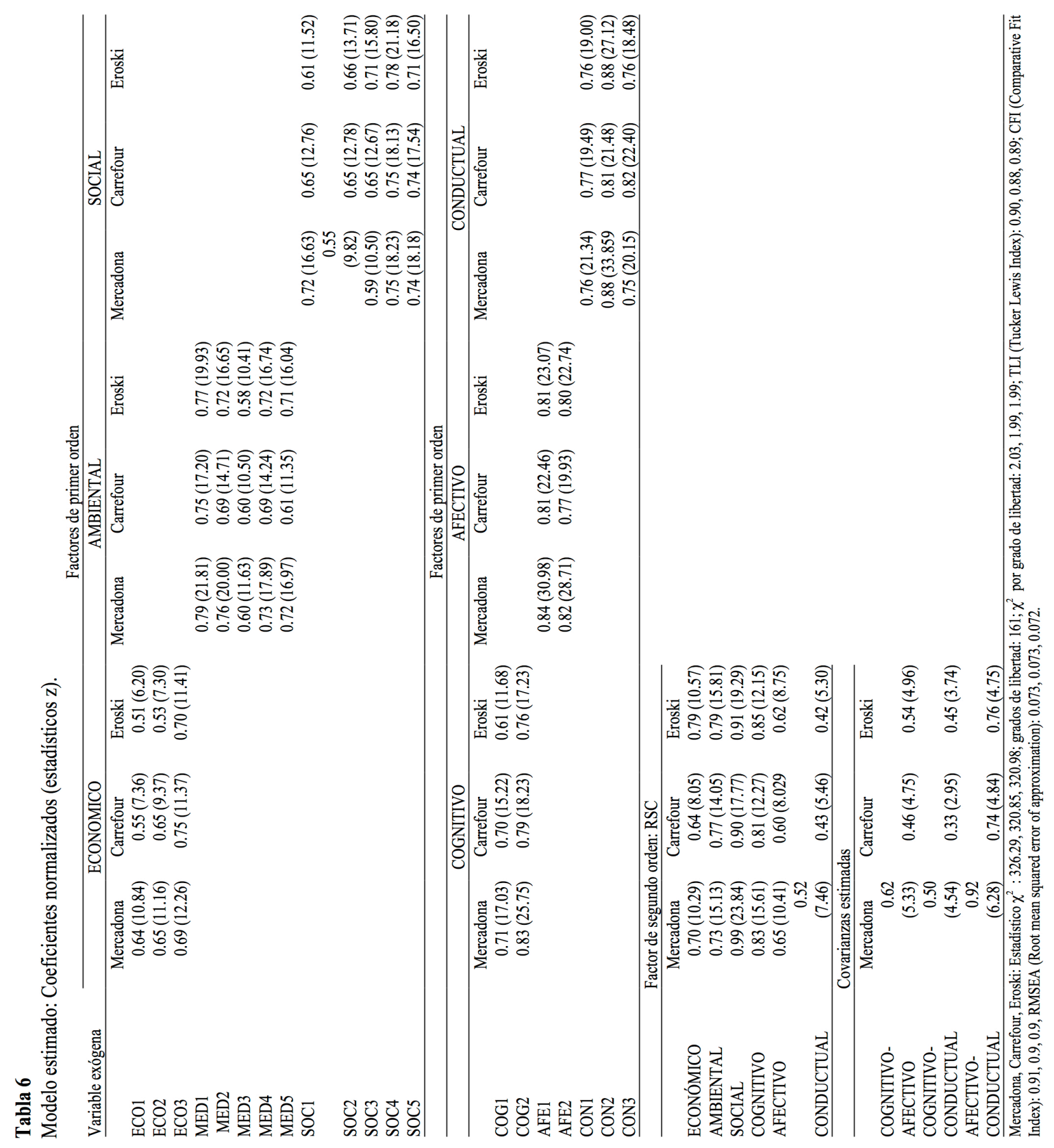


constructos de primer orden del modelo. Adicionalmente se ha calculado la matriz de cargas factoriales y cargas factoriales cruzadas, y se ha constatado que para cada constructo las cargas factoriales cruzadas son inferiores a las de cada indicador con su propio constructor (véase Tabla 5).

Por otra parte para contrastar las hipótesis relativas a la posible influencia de la RSC sobre la actitud del consumidor se ha construido un conjunto de tres factores de primer orden: cognitivo, afectivo y conductual. Estos tres factores no observados se miden a través de las variables observadas COG1, COG2 (cognitivo), AFE1, AFE2 (afectivo) y CON1, CON2 y CON3 (conductual). Las hipótesis de este trabajo H1, H2 y H3 se verificarán a través de los coeficientes estructurales del modelo que ligan a la RSC con los factores cognitivo, afectivo y conductual (véase Figura 3).

Los modelos se han estimado por separado para Mercadona, Carrefour y Eroski usando el método de máxima verosimilitud, y la matriz de varianzas y covarianzas se ha estimado a través de las matrices de información observadas (OIM) ofrecido por el software STATA 12, con los resultados que se presentan en la tabla 6 .

Los modelos ajustados presentan estadísticos de ajuste acordes con los estándares habituales. El estadístico $\chi^{2}$ por grado de libertad está en torno a 2 en los tres modelos ajustados y los estadísticos de ajuste de Tucker Lewis y CFI se encuentran en el entorno de 0,90. Por otro lado los coeficientes estimados son todos significativos al nivel del 5\% (véase Tabla 6).

\section{Conclusiones}

En este trabajo se ha utilizado una encuesta a una muestra de conveniencia de consumidores para medir la influencia de tres dimensiones diferentes de la RSC en la actitud del consumidor hacia tres empresas minoristas.

Se ha constatado una cierta estabilidad de la estructura dimensional estimada de la RSC en las tres empresas incluidas en la encuesta lo que pondría de manifiesto la validez del modelo. Por otra parte, no se han apreciado diferencias en función de si la empresa es o no familiar, lo cual puede deberse a que en los tres casos considerados se trata de empresas de gran tamaño.

En las tres empresas la dimensión percibida con mayor incidencia en la percepción de la RSC es la dimensión social seguida de la ambiental y la económica.

En la dimensión social las acciones que en las tres empresas presentan mayores cargas han sido: "Intenta tener prácticas igualitarias de oportunidades de empleo" y "Le preocupa la creación de puestos de trabajos". Por lo tanto, la percepción de la dimensión social a través de estas actividades es acorde a las preocupaciones actuales derivadas de las dificultades del mercado laboral, como consecuencia de la coyuntura económica. En la práctica estos resultados recomiendan a las empresas comunicar al mercado de manera clara y precisa sus políticas, acciones y resultados respecto a las cuestiones de la RSC que tienen una gran importancia en la percepción de que el consumidor tiene de la empresa.

Por otra parte, en la dimensión económica la acción que presenta mayor carga es "Le preocupa que los trabajadores estén bien remunerados" mientras que en la dimensión medioambiental lo es la acción "Patrocina actividades a favor del medioambiente". Aunque estas dimensiones tienen una menor influencia en la percepción de la RSC que la dimensión social, su impacto es también importante por lo que las empresas no deben descuidar comunicar al mercado sus políticas y resultados particularmente en las dos actividades señaladas.

En cuanto a la relación de la RSC con la actitud del consumidor, la investigación también corrobora claramente que las acciones de RSC influyen de manera directa y positiva en la actitud del consumidor, y pone de manifiesto diferencias entre los aspectos cognitivo, afectivo y conductual.

Si bien la influencia de la RSC sobre las tres dimensiones de la actitud es directa y positiva se observa que ésta tiene más fuerza en la componente cognitiva, y en menor medida en la afectiva y en la conductual. No obstante, la influencia positiva de las políticas de RSC sobre el comportamiento de compra del consumidor queda reflejada tanto en la relación directa de la RSC con la componente conductual como por la interrelación de las tres componentes, lo cuál refuerza la influencia de la RSC percibida en el comportamiento de compra a través de la componente cognitiva.

Sin embargo, estos resultados también revelan que si las empresas pretenden conseguir una mayor influencia de sus políticas de RSC en el comportamiento del consumidor deben identificar mejor cuáles son las acciones de RSC que más interesan a los consumidores y que en mayor medida 
les llevarían a incrementar sus compras; evidentemente, todo esto sin olvidar las acciones que influyen sobre la componente cognitiva y afectiva porque, como se ha puesto de manifiesto, las tres dimensiones están interrelacionadas.

En definitiva, los resultados han permitido contrastar el modelo teórico propuesto para medir la influencia de las acciones de RSC en la actitud del consumidor, sin que se hayan observado diferencias en función de la naturaleza de la empresa, lo que favorece la implantación de políticas de RSC en las grandes empresas de distribución minorista, al poner de manifiesto su impacto en el comportamiento de compra del consumidor.

No obstante, a pesar de que los resultados son consistentes con la percepción de las empresas incluidas en el análisis, según el ranking de Empresas Responsables (Merco, 2013), e incluso con otras recientes investigaciones en el sector minorista de alimentación de Estados Unidos (Ailawadi et al. 2011), las posibles limitaciones que se derivan de las características de la muestra utilizada aconsejan interpretar las conclusiones con las debidas cautelas, por lo que sería conveniente corroborar estos resultados con una muestra aleatoria de consumidores.

Por último, futuras investigaciones podrían profundizar en los resultados de este trabajo en diferentes líneas. En primer lugar, es importante mejorar la identificación de las actividades de RSC que tienen mayor influencia positiva sobre el comportamiento de compra del consumidor, de manera que los esfuerzos inversores de las empresas en sus políticas de RSC les permitan obtener un mayor retorno. En segundo lugar, para mejorar el conocimiento sobre la influencia de la RSC percibida en la actitud del consumidor se puede ahondar en la relación entre los componentes cognitivo, afectivo y conductual, incorporando al modelo una estructura de relaciones causales unidireccionales o bidireccionales entre ellas y la RSC. Tercero, puede ser importante analizar cómo influye la diferencia de enfoque de la RSC en cada empresa sobre la actitud del consumidor porque investigaciones anteriores han puesto de manifiesto que en este sector los grandes minoristas no están desarrollando las mismas políticas (Jones et. al, 2007). En este sentido, incluso podría considerarse la influencia de la velocidad de implantación de las prácticas de RSC (Baker et al., 2012). Cuarto, el modelo puede considerar relaciones indirectas a través de variables mediadoras como son: precio, surtido, marca propia, localización, imagen, identificación con la enseña, etc. En este sentido, cabe destacar la posible incidencia de la estrategia de comunicación de la RSC. Varios autores han demostrado que la comunicación de la $\mathrm{RSC}$ es una buena estrategia para promover la identificación con la empresa (Lichtenstein et al., 2004; Marín y Ruiz, 2007). Quinto, la RSC está vinculada a diferentes grupos de interés (Balmer et al., 2007 y Hildebrand et al., 2011), por lo que sería interesante comprender $\mathrm{y}$ entender el comportamiento ante la RSC, entre otros, de los empleados, inversores y proveedores para seguir así la línea del marketing corporativo (Balmer et al., 2009) y su influencia en el comportamiento del consumidor. Finalmente, en esta investigación se ha analizado la influencia de la RSC en la actitud del consumidor de la gran empresa comercial minorista de alimentación. Futuras investigaciones pueden ampliar esta investigación a otros sectores e industrias.

\section{Bibliografía}

Adams, R.J. (2002). Retail profitability and sweatshops: a global dilemma. Journal of Retailing and Consumer Services, 9, 147-153.

Ailawadi, K. L., Luan, Y. J., Neslin, S. A. \& Taylor, G. A. (2011). The Impact of Retailers' Corporate Social Responsibility on Price Fairness Perceptions and Loyalty. Working Paper, Tuck School of Business, Dartmouth College. Disponible en: http://idei.fr/doc/conf/inra/2011/ailawadi\%20kusum.pdf (acceso 1 agosto 2013).

Alonso, J; Grande, I. (2010). Comportamiento del Consumidor. Madrid: Ediciones ESIC.

Anderson, W., Fornell, C. \& Mazvancheryl, S.K. (2004). Customer Satisfaction and Shareholder Value. Journal of Marketing, 68, 172-285.

Anselmsson, J. \& Johansson, U. (2007). Corporate social responsibility and the positioning of grocery brands. An exploratory study of retailer and manufacturer brand at point of purchase. International Journal of Retail \& Distribution Management, 35(10), 835-856.

Arnett, D., German, S \& Hunt, S. (2003). The Identity Salience Model of Relationship Marketing Success: The Case of Nonprofit Marketing. Journal of Marketing, 67, 89-105.

Arnold, S.J., Handelman, J.M. \& Tigert, D.J. (1996). Organizational legitimacy and retail store patronage. Journal of Business Research, 35, 229-239.

Auger, P. \& Devinney, T.M. (2007). Do what consumers say matter? The misalignment of preferences with unconstrained ethical intentions. Journal of Business Ethics, 76(4), 361-83.

Bagozzi, R.P. (1997). Goal-directed Behaviors in 
Marketing: The Role of Emotion, Volition, and Motivation. Psychology \& Marketing, 4(3), 309-313.

Bagozzi, R.P. \& Dholakia, U. (1999). Goal setting and goal striving in consumer behavior. Journal of Marketing, 63, 19-32.

Bagozzi, R.P., Tybout, A.M., Craig, C.S. \& Sterntha, B. (1979). The Construct Validity of the Tripartite Classification of Attitudes. Journal of Marketing Research, 16(1), 88-95.

Baker, C.R., Cohanier, B. \& Pederzoli, D. (2012). Corporate Social And Environmental Reporting In The Large Retail Distribution Sector. Procedia Economics and Finance, 2, 209-218.

Balmer, J.M.T. (2011). Corporate marketing myopia and the inexorable rise of a corporate marketing logic: perspectives from identity-based views of the firm. European Journal of Marketing, 45(9/10), 1329-1352.

Balmer, J.M.T., Fukukawa, K. \& Gray, E.R. (2007). The nature and management of ethical corporate identity: a commentary on corporate identity, corporate social responsibility and ethics. Journal of Business Ethics, 76(1), 7-15.

Balmer, J.M.T., Stuart, H. \& Greyser, S.A. (2009). Aligning identity and strategy: corporate branding at British Airways in the late twentieth century. California Management Review, 51(3), 6-23.

Barin, L. \& Michael, D. (2010). How do Leading Retail MNCs Leverage CSR Globally? Insights from Brazil. Journal of Business Ethics, Springer, 243-263.

Barone, M.J., Miyazaki, A.D. \& Taylor, K.A. (2000). The Influence of Cause-Related Marketing on Consumer Choice: Does One Good Turn Deserve Another? Journal of the Academy of Marketing Science, 28, 248-263.

Becker-Olsen, K.L., Cudmore, B.A. \& Hill, R.P. (2006). The impact of perceived corporate social responsibility on consumer behaviour. Journal of Business Research, 59(1), 46-53.

Berens, G., Van Riel, C.B.M. \& Van Bruggen, G.H. (2005). Corporate Associations and Consumer Product Responses: The Moderating Role of Corporate Brand Dominance. Journal of Marketing, 69, 35-48.

Bergami, M. \& Bagozzi, R.(2000). Selfcategorization, affective commitment and group selfesteem as distinct aspects of social identity in the organization. British Journal of Social Psychology, 39(1), $555-577$.

Berry, 1.1. (2000). Cultivating service brand equity. Journal of the Academy of Marketing Science, 28, 128137.

Bhattacharya, C.B., Korschun, D. \& Sen, S. (2008). Strengthening stakeholder-company relationships through mutually beneficial corporate social responsibility initiatives. Journal of Business Ethics, 85(2), 257-272.
Bhattacharya, C.B. \& Sen, S. (2003). ConsumerCompany Identification: A Framework for Understanding Consumers' Relationship With Companies. Journal of Marketing, 67(4), 76-88.

Bigné, E., Alvarado, A., Aldás, J. \& Currás, R. (2011). Efectos de la responsabilidad social corporativa percibida por el consumidor sobre el valor y la satisfacción con el servicio. Revista Europea de Dirección y Economía de la Empresa, 20(4), 139-160.

Bigné, E., Andreu, L., Chumpitaz, R. \& Swaen, V. (2006). La influencia de la responsabilidad de la responsabilidad social corporativa en el comportamiento de compra de estudiantes universitarios. ESIC Market, 6, 163-189.

Bigné, E., Chumpitaz, R., Andreu, L. \& Swaen, V. (2005). Percepción de la Responsabilidad Social Corporativa: Un análisis corss-cultural. Universia Business Review, 5, 14-27.

Bigné, E. \& Currás, R. (2008). ¿Influye la imagen de responsabilidad social en la intención de compra? El papel de la identificación del consumidor con la empresa. Universia Business Review, 19, 10-23.

Bigné, E., Currás, R. \& Aldás,J. (2012). Dual nature of cause-brand fit: Influence on corporate social responsibility consumer perception. European Journal of Marketing, 46(3/4), 575-594.

Boal, K.B. \& Peery, N. (1986). The cognitive structure of corporate social responsibility. Journal of Management, 11(3), 71-83.

Brammer, S. \& Millington, A. (2005). Firm, size, Organizational Visibility and Corporate Philanthropy: An Empiical Analysis. Business Ethics: A European Review, 15(1), 6-8.

Brown, T. (1998). Corporate Associations in Marketing: Antecedents and Consequences. Corporate Reputation Review, 1(3), 215-233.

Brown, T.J. \& Dacin, P.A. (1997). The company and the product: Corporate Associations and Consumer Product Respones. Journal of Marketing, 61(1), 68-84.

Campbell, J. L. (2007). Why would corporations behave in socially responsible ways? Institutional theory of corporate social responsibility. Academy of Management Review, 32(3), 946-967.

Carrero, I. \& Valor, C. (2012). CSR-labelled products in retailer's assortment. A comparative study of British and Spanish retailers. International Journal of Retail \& Distribution Management, 40(8), 629-652.

Carrigan, M. \& Attalla, A. (2001),. The myth of the ethical consumer - do ethics matter in purchase behaviour? Journal of Consumer Marketing, 18(7), 56078 .

Carroll, A.B. (1999). Corporate social responsibility: evolution of a definitional construct. Business \& Society, $38(3), 268-295$. 
Chin, W.W. \& Tood, P.A. (1995). On the Use, Usefulness, and Ease of Use of Structural Equation Modeling in MIS Research: A Note of Caution. MIS Quarterly, 19(2), 237-246.

CONE (2002). Cone corporate citizenship study. The role of cause branding. Executive Summary, CONE, Boston, Massachussets. http://www.coneinc.com

Connor, J. O. (2001). Global price fixing: Our customers are the enemy (studies in industrial organization). Berlin: Springer.

Creyer, E.H. \& Ross, W.T.J. (1997). The influence of firm behavior on parchase intention: do consumers really care about business ethics? The Journal of Consumer Marketing, 14(6), 421-432.

Cronin, J.J., Brady, M.K. \& Hult, G. (2000). Assessing the Effects of Quality, Value, and Customer Satisfaction on Consumer Behavioral Intentions in Service Environments. Journal of Retailing, 76(2), 193218.

Deloitte. (2013). Global Powers of Retailing 2013 Retail Beyond. Disponible en: http://www.deloitte.com/assets/Dcom-

Australia/Local\%20Assets/Documents/Industries/Consum er\%20business/Deloitte_Global_Powers_of_Retail_2013. pdf (Acceso 3 marzo 2014)

Drumwright, M.(1994). Socially Responsible Organizational Buying: Environmental Buying as a Noneconomic Buying Criterion, Journal of Marketing, 58(3), 1-19.

Du, S., Bhattacharya, C.B. \& Sen, S. (2007). Reaping Relational Rewards from Corporate Social Responsibility: The Role of Competitive Positioning. International Journal of Research in Marketing, 24(3), 224-241.

Dyer, W. G. (1988). Culture and Continuity in Family Firms. Family Business Review, 1(1), 37-50.

Elkington, J. (1997). Cannibals with Forks: The Triple Bottom Line of 21 st Century Business. Capstone, Oxford.

Elkington, J. (1998). Accounting for the triple Bottom Line. Measuring Business Excellence, 2(3), 18-22.

Fan, Y. (2005). Ethical branding and corporate reputation. Corporate Communications An International Journal, 10(4), 341-350.

Fassin, Y., van Rossem, A. \& Buelens, M. (2011). Small-Business Owner-Managers' Perceptions of Business Ethics and CSR-Related Concepts. Journal of Business Ethics, 98(3), 425-453.

Fernández, D. \& Merino, A. (2005). ¿Existe disponibilidad a pagar por responsabilidad social corporativa? Percepción de los consumidores. Universia Business Review, III trimestre, 38-53.

Fernández, P. (2013). La evolución de los mayores negocios familiares en España (1964-2010). Nuevas fuentes, nuevos métodos, nuevos resultados. Asociación
Española de Historia Económica, Documento de Trabajo $\mathrm{n}^{\mathrm{o}} \quad 1307, \quad 1-39$. Disponible en: http://www.aehe.net/2013/06/dt-aehe-1307.pdf (Acceso 3 marzo 2014).

Fornell, C. \& Larker, D.F. (1981). Structural Equation Models with Unobservable Variables and Measurement Error. Journal of Marketing Research, 18(1), 39-50.

Fullerton, G. (2003). When Does Commitment Lead to Loyalty? Journal of Service Research, 5(4), 333-344.

García de los Salmones, M.M., Herrero, A. \& Rodríguez del Bosque, I. (2005). Influence Influence of Corporate Social Responsibility on Loyalti and Valuation of Services. Journal of Business Ethics, 61, 369-385.

Garriga, E. \& Melé, D. (2004). Corporate Social Responsibility Theories: Mapping the Territory. Journal of Business Ethcis, 53(1/2), 51-71.

Göbbels, M. (2002). Reframing Corporate Social Responsibility: The Contemporary Conception of a Fuzzy Notion. Journal of Business Ethics, 44, 95-105.

Gourville, J.T. \& Rangan, V.K. (2004). Valuing the Cause Marketing Relationship. California Management Review, 47(1), 38-58.

Green, T. \& Peloza, J. (2011). How Does Corporate Social Responsibility Create Value for Consumers? Journal of Consumer Research, 28(1), 48-56.

Grönroos, C. (1994). From Marketing Mix to Relationship Marketing. Towards a Paradigm Shift in Marketing. Management Decision, 32 (2), 4-32.

Grunert, K.G. (2005). Food quality and safety: consumer perception and demand. European Review of Agricultural Economics, 32(3), 369-91.

Gupta, S. \& Pirsch, J. (2008). The influence of a retailer's corporate social responsibility porgram on reconceptualizing store image. Journal of retailing and consumer services, 15(6), 516-526.

Guiry, M., Mägi, A.W. \& Lutz, R.J. (2006). Defining and measuring recreational shopper identity. Journal of the Academy of Marketing Science, 34(1), 74-83.

Gürhan-Canli, Z. \& Batra, R. (2004). When Corporate Image Affects Product Evaluations: The Moderating Role of Perceived Risk. Journal of Marketing Research,, 41, 197-205.

Hair, J.F., Black, W.C., Babin, B.J. \& Anderson, R.E. (2010). Multivariate Data Analysis, 7th ed. New Jersey: Pearson Prentice Hall.

Hall, A. \& M. Nordqvist (2008). Professional Management in Family Businesses: Toward an Extended Understanding. Family Business Review, 21(1), 51-69.

Handelman, J.M. \& Arnold, S.J. (1999). The role of marketing actions with a social dimension: appeals to the institutional environment. Journal of Marketing, 63, 3348. 
Harwood, I., Humby, S. \& Harwood, A (2011). On the resilence of Corporate Social Responsibility. European Management Journal, 29, 283-290.

Hildebrand,D., Sen, S. \& Bhattacharya, C.B. (2011). Corprate social responsibilty: a corporate marketing perspective. European Journal of Marketing, 45( 9/10), 1353-1364.

Holt, D. B., Quelch, J. A. \& Taylor, E. L. (2004). How global brands compete. Harvard Business Review, 82(9), 68-75.

Indisa (2011). Anuario de la distribución 2011-2012. Información y Distribución Anual, Madrid.

IPSOS (2011). Key Audience Research, Madrid.

Jamali, D., Zanhour, M. \& Keshishian, T. (2009). Peculiar Strengths and Relational Attributes of SMEs in the Context of CSR. Journal of Business Ethics, 87, 355377 .

Jenkins, H. (2006). Small Business Champions for Corporate Social Responsibility. Journal of Business Ethics, 67, 241-256.

Jeurissen, R. (2000). John Elkington, Cannibals With Forks: The Triple Bottom Line of 21st. Journal of Business Ethics, 23(2), 229-231.

Jones, P., Comfort, D. \& Hillier, D. (2007). Corporate Corporate social responsibility: a case study of the top ten global retailers. Journal of Business, 2(1), 2335 .

Kamins, M. \& Folkes, V. (1999). Effects of information about firms' ethical and unethical actions on consumer attitudes. Journal of Consumer Psychology, 8(3), 243-259.

Keller, K.L. (1993). Conceptualizing, Measuring and Managing Customer-Based Brand Equity, Journal of Marketing, 56, 1-22.

Kilcullen, M. \& Kooistra, J.O. (1999). At least do no harm: sources on the changing role of business ethics and corporate social responsibility. Reference Services Review, 27 (2), 158-178.

Klein, J. \& Dawar, N. (2004). Corporate Social Responsibility and Consumers' Attributions and Brand Evaluations in a Product-Harm Crisis. International Journal of Research in Marketing, 21(3), 203-217.

Ko, E., Hwang, Y.K. \& Kim, E.Y. (2013). Green marketing' functions in building corporate image int the retail setting. Journal of Business Research, 66(10), 17091715 .

Krech, D., Crutchfield, R. \& Ballachey, E. (1962). Individuals in Society. New York: McGraw-Hill Book Company.

Laroche, M., Ueltschy, L.C., Abe, S., Cleveland, M. \& Yannopoulos, P.P. (2004). Service quality perceptions and customer satisfaction: evaluating the role of culture. Journal of International Marketing, 12(3), 58-85.
Lichtenstein, D.R., Drumwright, M.E. \& Braig, B.M. (2004). The Effect of Corporate Social Responsibility on Customer Donations to Corporate-Supported Nonprofits. Journal of Marketing, 68, 16-63.

Luo X. \& Bhattacharya, C.B. (2006). Corporate Social Responsibility, Customer Satisfaction and Market Value. Journal of Marketing, 70, 1-18.

Mackenzie, S., Podsakoff, P. \& Burke, C. (2001). The Problem of Measurement Model Misspecification in Behavioral and Organizational Research and Some Recommended Solutions. Journal of Applied Psychology, 90(4), 710-730.

Maignan, I. (2001). Consumers' Perceptions of Corporate Social Responsibilities: A Cross-Cultural Comparison. Journal of Business Ethics, 30, 57-72.

Maignan, I. \& Ferrell, O.C. (2004). Corporate social responsibility and marketing: an integrative framework. Journal of the Academy of Marketing Science, 32(1), 319 .

Maignan, I., Ferrell, O.C. \& Ferrell, L (2005). A stakeholder model for implementing social responsibility in marketing. European Journal of Marketing, 39(9/10), 956-977.

Maignan, I., Ferrell, O.C. \& Hult, G. (1999). Corporate citizenship: Cultural antecedents and business benefits. Journal of the Academy of Marketing Science, 27(4), 455-469.

Marín, L. \& Ruiz, S. (2007). I Need You Too! Corporate Identity Attractiveness for Consumers and the Role of Social Responsibility. Journal of Business Ethics, $71,245-260$.

Massa, S. \& Testa, S. (2012). The role of ideology in brand strategy: the case of a food retail company in Italy. International Journal of Retail \& Distribution Management, 40(2), 109-127.

Mathieson, K., Peacock, E. \& Chin, W.W. (2001). Extending the Technology Acceptance Model: The Influence of Perceived User Resources. The DATA BASE for Advances in Information Systems, 32(3), 86112.

Menon, S. \& Kahn, B. (2003). Corporate sponsorships of philanthropic activities: when do they impact perception of sponsor brand? Journal of Consumer Psychology, 13(3), 316-327.

Mercadona (2014). Memoria Anual Mercadona 2013. Disponible en: www.mercadona.es/corp/esphtml/noticias. (Acceso 5 marzo 2014)

Merco (2013). Monitor Empresarial de Reputación Corporativa. Disponible en: http://www.merco.info/es/countries/4/rankings/14 (acceso 1 agosto 2013).

Miller, D., Miller , I. \& Scholnick, B. (2008). Stewardship vs Stagnation: An Empirical Comparison of Small Family and Non Family Businesses. Journal of 
Management Studies, 45(1), 51-78.

Morgeson, F.V., Mithas, S., Keiningham, T.L. \& Aksoy, L. (2011). An investigation of the cross-national determinants of customer satisfaction. Journal of the Academy of Marketing Science, 39(2), 198-215.

MORI (2000). European attitudes towards corporate citizenships, Corporate social responsibiltiy update 2 . Market \& Opinion Research International, http://www.mori.com

Murillo, D. \& Lozano, J. (2006) SMEs and CSR: An Approach to CSR in Their Own Words. Journal of Business Ethics, 67, 227-240.

Nicholls, A.J. (2002). Strategic options in fair trade retailing. International Journal of Retail and Distribution Management, 30(1), 6-17.

Norman, W. \& MacDonald, C. (2004). Getting to the Bottom of "Triple Bottom Line, Business Ethics Quarterly. 14(2), 243-262.

Oliver, R.L., Rust, R.T. \& Varki, S. (1997). Customer Delight: Foundations, Findings, and Managerial Insight. Journal of Retailing, 73(3), 311-336.

Opinea (2013). Las marcas reconocidas por sus acciones de RSC. Disponible en: http://www.opinea.es/actualidad/las-marcas-masreconocidas-por-sus-acciones-de-rsc-2/ (Acceso 3 marzo 2014).

Oppewal, H., Alexander, A. \& Sullivan, P. (2006). Consumer perceptions of corporate social responsibility in town shopping centres and their influence on shopping evaluations. Journal of Retailing and Consumer Services, $13,261-274$.

Ou, W.-M., Abratt, R. (2006). Diagnosing the relationship between corporate reputation and retail patronage. Corporate Reputation Review, 9 (4), 243-257.

Peloza, J. \& Shang, J. (2011). How can corporate social responsibility activities create value for stakeholders? A systematic review. Journal of Academy of Marketing Science, 39, 117-135.

Pérez, A., García de los Salmones, M.M. \& Rodríguez del Bosque , I. (2012). The effect of Corporate Associations on Consumer Behaviour. European Journal of Marketing, 47(1), 218-238.

Pérez, A. \& Rodríguez del Bosque, I. (2012). La imagen de Responsabilidad Social Corporativa en un contexto de crisis económica: El caso del sector financiero en España. Universia Business Review, I trimestre, 14-29.

Porter, M. \& Kramer, M. (2011). The big idea: Created shared value. How to reivent capitalism and unleash a wave of innovation and growth. Harvard Business Review, 1-16.

Powell, S.M. (2011). The nexus between ethical corporate marketing, ethical corporate, corporate identity and corporate social responsibility. An internal organisational perspective. European Journal of Marketing, 45(9/10), 1365-1379.

Prout, J. (2006). Corporate responsibility in the global economy: A business case. Society and Business Review, 1(2), 184-191.

Putrevu, S. \& Lord, K (1994). Comparative and noncomparative advertising: attitudinal effects under cognitive and affective involvement conditions. Journal of Advertising, 23, 77-91.

Reichheld, F.F. (2003). The one number you need to grow. Harvard Business Review, 81(12), 46-54.

Rivera, J., Arellano,R. \& Molero, V.M. (2009). Conducta del consumidor. Estrategias y políticas aplicadas al marketing. Madrid: Ediciones ESIC.

Rosenberg, M. \& Hovland, C. (1960). Cognitive, Affective and Behavioral Components of Attitudes. New Haven, Connecticut: Yale University Press.

Savitz, A. (2013). The triple bottom line: how today's best-run companies are achieving economic, social and environmental success-and how you can too. John Wiley \& Sons.

Secretaría de Estado de Comercio. (2011). Análisis por formatos y subsectores. La distribución comercial en España en 2010. Boletín Económico de ICE, 3015, 99$126 . \quad$ Disponible en: http://www.revistasice.com/CachePDF/BICE 3015 99126_33B6BB795F3CFBE6C08E02FBBA4200F9.pdf (Acceso 3 marzo 2014)

Sen,S. \& Bhattacharya, C.B. (2001). Does doing good always lead to doing better? Consumer reactions to corporate social responsibility. Journal of Marketing Research, 38(5), 225-243.

Sen, S., Bhattacharya, C.B., \& Korschun, D. (2006). The Role of Corporate Social Responsability in Strengthening Multiple Stakeholder Relationships: A field experiment. Journal of the Academy of Marketing Science, 38,(2), 158-166.

Sirgy, M.J., Johar, J.S., Samli, A.C. \& Claiborne, C.B. (1991). Self-congruity versus functional congruity: predictors of consumer behaviour. Journal of the Academy of Marketing Science, 19(4), 363-375.

Simon, F.L. (1995). Global corporate philanthropy: a strategic framework. International Marketing Review, 12(4), 20-38.

Slaper, T.F. \% Hall, T.J. (2011). The triple bottom line: what is it and how does it work? The Indiana Business Review, 86(1), 4-8.

Söderlund, M. (2002). Customer Familiarity and Its Effects on Satisfaction and Behavioral Intentions. Psychology \& Management, 19(10), 861-879.

Swaen, J.E. \& Trawick, I.F. (1981). Disconfirmation 
of Expectations and Satisfaction with a Retail Service. Journal of Retailing, 57(3), 49-67.

Taylor, S.A., Hunter, G.L. \& Lindberg, D.L. (2007). Understanding (customer-based) brand equity in financial services. Journal of Services Marketing, 21(4), 241-252.

Torres, A., Bijmolt, T.H.A., Tribó, J.A. \& Verhoef, P. (2012). Generating global brand equity through corporate social responsibility to key stakeholders. International Journal of Research in Marketing, 29, 13-24.

Van Marrewijk, M. (2003). Concepts and definitions of CSR and corporate sustainability: Between agency and communion. Journal of Business Ethics, 44(2/3), 95-105.

Vives, A. (2006). Social and Environmental Responsibility in Small and Medium Enterprises in Latin America. The Journal of Corporate Citizenship, 21, 3950 .

Yoon, Y., Gürhan-Canli, Z. \& Schwarz, N. (2006). The effect of corporate social responsibility activities on companies with bad reputations. Journal of Consumer Psychology, 16(4), 377-390.

Wagner, T., Bicen, P. \& Hall, Z.R.(2008). The dark side of retailing: towards a scale of corporate social irresponsibility. International Journal of Retail \& Distribution Management, 36(2), 124-142.

Walsh, G. \& Bartikowski, B. (2013), Exploring corporate ability and social responsibility associations as antecedents of customer satisfaction cross-culturally. Journal of Business Research, 66, 989-995.

Wan, H. \& Schell, R. (2007). Reassessing corporate image - an examination of how image bridges symbolic relationships with behavioral relationships. Journal of Public Relations Research, 19, 25-45.

Wheeler, D. \& Elkington, J. (2001). The end of the corporate environmental report? Or the advent of cybernetic sustainability reporting and communication. Business Strategy and the Environment, 10(1), 1-14.

Wirtz, J. \& Bateson, J.E.G. (1999). The Dimensionality of Consumption Emotion Patterns and Consumer Satisfaction. Journal of Consumer Research, 18(1), 84-91.

Wuu, B.T.W. \& Petroshius, S.M. (1987). The halo effect in store image management, Academy of Marketing Science Journal, 15(3), 44-51.

Zhang, J., Beatty, S.E. \& Walsh, G. (2008). Review and future directions of cross-cultural consumer services research. Journal of Business Research, 61(3), 211-24.

Zeithaml, V.A., Berry L.L. \& Parasuraman, A. (1996). The Behavioural Consequences of Service Quality. Journal of Marketing, 60, 31-46. 